\title{
杂环化试剂与烯烃快速组装构建氮杂芳烃
}

\author{
欧阳旋慧李金恒*
}

(南昌航空大学 江西省持久性污染物控制与资源循环利用重点实验室 南昌 330063)

\section{Heterocyclization Reagents for Rapid Assembly of N-Fused Het- eroarenes from Alkenes}

\author{
Ouyang, Xuanhui \\ Li, Jinheng* \\ (Key Laboratory of Jiangxi Province for Persistent Pollutants Control and Resources Recycle, \\ Nanchang Hangkong University, Nanchang 330063)
}

氮杂芳烃是一类十分重要的药物骨架, 广泛地存在 于天然产物和药物分子中 ${ }^{[1]}$. 由于其具有改善生物活性 的特点 ${ }^{[2]}$, 氮杂芳烃已成为全球药品销售 TOP 200 中十 分常见的骨架(图 1). 相关大数据分析表明杂环的形成 是过去几十年来药物化学中最常用的反应之一 ${ }^{[3]}$. 同时, 由于氮杂芳烃特殊的光物理和电子传输性能, 在有机光 电材料领域也引起了广泛关注 ${ }^{[4]}$. 因此, 许许多多的有 机化学家付出了巨大努力, 尝试开发新的合成氮杂芳烃 方法 ${ }^{[-6]}$. 然而, 这些方法的应用很大程度上受限于复杂 的合成前体或繁琐的合成路线. 目前关于杂环的知识仍 然有很大的不足, Pitt 等 ${ }^{[7]}$ 列举了一个总共 24847 个杂芳 烃的表, 其中只有少于 $10 \%$ 的杂芳烃被人工合成出来. 基于杂芳环的合成现状, 利用简单易得的原料或试剂实 现杂芳环的有效合成仍然是杂芳环合成的重点, 同时也 必将打开新时代新药和新材料创制的大门.

烯烃是简单易得的大宗化学品, 在许多领域被广泛 用作合成原料 ${ }^{[8]}$. 合理设计易于与各种烯烃反应的新型 杂环化试剂, 为快速组装具有高度多样性的氮杂芳烃提 供了实用的策略. 近年来, 苏州大学材料与化学化工学 部朱晨课题组 ${ }^{[9]}$ 发展了一系列基于氮杂环砜类的烯烃官 能团化试剂. 其中, 当氮杂环试剂另一侧的烷基带有离 去基团/原子时, 该官能团试剂能通过官能团迁移实现 烯烃双官能化反应和卤化反应等.

该研究团队设想将烯烃双官能化反应和后期环化 反应的协同一锅法应用于杂芳烃合成, 其中包含去基 团/原子等过程, 实现了由简单易得的烯烃高效构建氮 杂芳烃(Scheme 1). 该策略经过一系列合理设计和制备<smiles>Cc1nnc2n1-c1ccc(Cl)cc1C(c1ccccc1)NC2</smiles>

Alprazolam<smiles>O=c1ccnc2[nH][nH]cc1-2</smiles>

Allopurinol<smiles>Cc1ccc2c(c1)Nc1sc(C)cc1C(N1CCN(C)CC1)=N2</smiles>

Olanzapine<smiles>Clc1ccc2nsnc2c1NC1=NCCN1</smiles>

Tizanidine

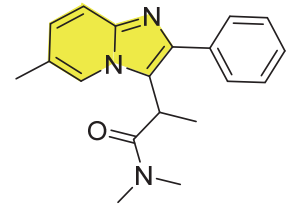

Zolpidem<smiles>N[C@@H](CC(=O)N1CCn2c(nnc2C(F)(F)F)C1)Cc1cc(F)c(F)cc1F</smiles><smiles>CCOC(=O)OCCn1nc2ccccn2c1=O</smiles>

图 1 全球药品销售 TOP 200 中氮杂芳烃举例

Figure 1 Selected examples containing $N$-fused heterocycles in top 200 pharmaceuticals

杂环化试剂, 经过多步串联环化反应，合成出大量不同 类型的氮杂芳烃, 其中包括了吲哚嗪等七种类型杂环骨 架.

* Corresponding author. E-mail: jhli@hnu.edu.cn. Published online August 22, 2021.. 


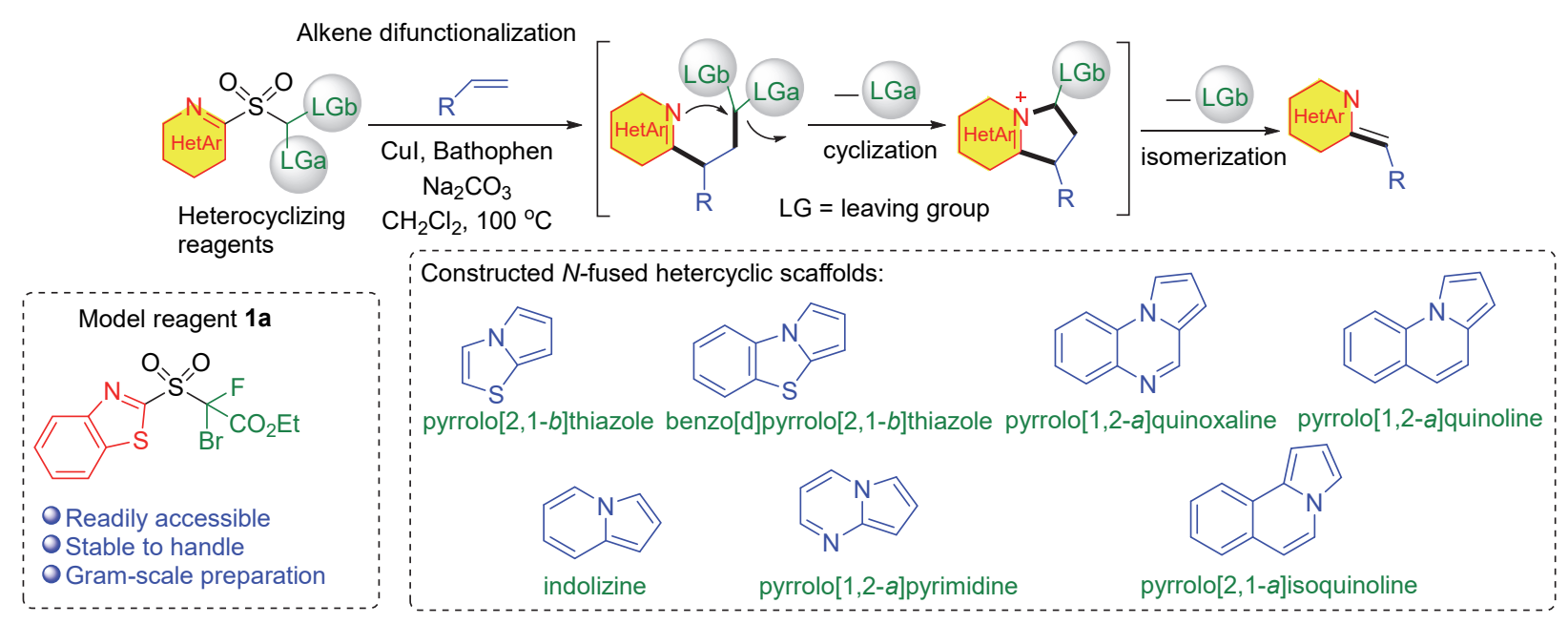

图式 1 杂原子环化试剂与烯烃快速构建氮杂芳烃

Scheme 1 Heterocyclization reagents for rapid assembly of $N$-fused heteroarenes from alkenes

该方法反应条件简单、温和、产率高、化学选择性 好，对不同类型的烯烃都有很好的兼容性，同时具有广 泛的官能团兼容性和产品多样性, 并为杂环芳烃的后期 修饰提供了实用的方法. 该研究团队也实现了复杂的天 然产物和药物的后期修饰以及官能团化 (图 2), 如香豆 素(Coumarin)、非布司他(Febuxostat)、非诺贝特(Fenofibrate)、卵磷脂(Follestrol)、二氢胆固醇(Dihydrocholesterol)、雄酮(Androstrone)、依折麦布(Ezetimibe). 同 时该方法可以在不影响产率的情况下实现克级反应, 表 明该方法在药物开发中的潜在用途.

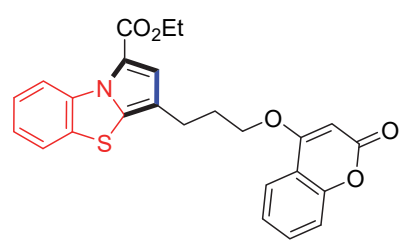

from Coumarin, $52 \%$

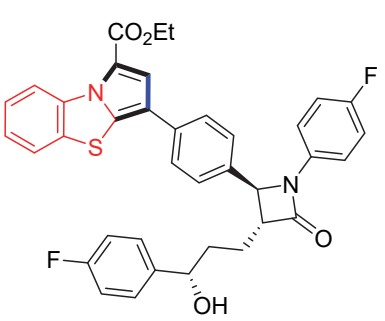

from Ezetimibe, $56 \%$

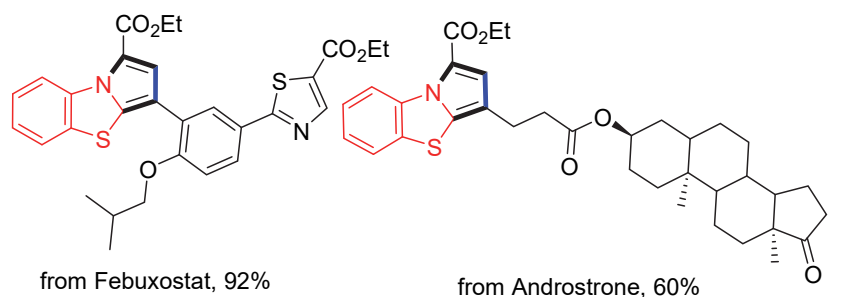

图 2 复杂氮杂芳烃的构建

Figure 2 Construction of heterocycles bearing complex scaffolds

机理研究表明, 该反应很可能经过铜催化剂与氮杂
环砜试剂中的 $\mathrm{C}-\mathrm{Br}$ 键之间的单电子转移(single electron transfer, SET), 生成自由基中间体, 并与烯烃加成, 然后发生杂芳基迁移和 $\mathrm{SO}_{2}$ 释放, 得到新的自由基. 该 自由基㩖取卤素生成双官能团化产物. 随后, 杂芳烃的 $\mathrm{N}$ 原子分子内亲核进攻卤原子, 再经过异构化和 HF 消 除去质子化过程, 最终得到多样性的氮杂芳烃. 朱晨课 题组基于氮杂环砜试剂, 发展了杂环化试剂与烯烃串联 环化反应，实现了氮杂芳烃的高效构建. 该方法成功实 现了天然产物和药物衍生物的后期修饰和官能团化, 并 且有望在药物化学和新材料合成研究等领域发挥作用.

该方法的创新点在于，在铜催化下利用多反应活性 位点试剂, 以烯烃为自由基链式(接力)桥, 实现中性氧 化还原自由基链式(接力)串联反应, 从而为发展新的自 由基串联反应提供了新思路, 即通过试剂理性设计, 可 以实现自由基反应革新.

\section{References}

[1] Kalinin, A. A.; Mamedov, V. A. Chem. Heterocycl. Compd. 2011, $46,1423$.

[2] McGrath, N. A.; Brichacek, M.; Njardarson, J. T. J. Chem. Educ. 2010, 87, 1348.

[3] Roughley, S. D.; Jordan, A. M. J. Med. Chem. 2011, 54, 3451.

[4] Wan, J.; Zheng, C.-J.; Fung, M.-K.; Liu, X.-K.; Lee, C.-S.; Zhang, X.-H. J. Mater. Chem. 2012, 22, 4502.

[5] Wang, B.; Liu, W.; Hu, H. Chin. J. Chem. 2006, 24, 279.

[6] Wang, D. C.; Xie, M. S.; Guo, H. M.; Qu, G. R.; Zhang, M. C.; You, S. L. Angew. Chem., Int. Ed. 2016, 55, 14111.

[7] Pitt, W. R.; Parry, D. M.; Perry, B. G.; Groom, C. R. J. Med. Chem. 2009, 52, 2952.

[8] Pintauer, T.; Matyjaszewski, K. Chem. Soc. Rev. 2008, 37, 1087.

[9] Wu, X.; Zhu, C. Acc. Chem. Res. 2020, 53, 1620. 